\title{
Avaliação da cobertura da Atenção Básica na detecção de adultos com diabetes e hipertensão
}

\author{
Evaluation of the coverage of Primary Health Care in detecting adults \\ with diabetes and hypertension
}

Bárbara Radigonda1, Regina Kazue Tanno de Souza $\mathbf{2}$, Luiz Cordoni Junior ${ }^{\mathbf{3}}$

RESUMO O presente estudo documental avaliou a detecção das pessoas com hipertensão e/ ou diabetes pelas Unidades Básicas de Saúde (UBS), identificadas em estudo de base populacional. Entre os 606 indivíduos incluídos, 386 (63,7\%) apresentaram registro de hipertensão e/ou diabetes na Ficha A, no Sishiperdia ou nos prontuários. A frequência de detecção foi significativamente mais elevada entre as pessoas de pior estrato econômico, que não exerciam atividade ocupacional e utilizavam a UBS. Os resultados mostram cobertura regular, predominantemente dos que utilizam os serviços e apontam necessidade de estratégias que ampliem o reconhecimento da população-alvo da atividade programática.

PALAVRAS-CHAVE Avaliação em saúde; Serviços de saúde; Atenção Primária à Saúde; Diabetes mellitus; Hipertensão.

ABSTRACT This documentary study evaluated the detection of people with hypertension and/or diabetes by Basic Health Units (UBS), identified in a populational based study. Among the included 606 individuals, 386 (63.7\%) of them had a record of hypertension and/or diabetes on Record $A$, in Sishiperdia or in the charts of UBS. The detection frequency was significantly higher among people with a worse economic stratum, who did not have an occupation and used the UBS. The results show regular coverage, predominantly those who use the services and point to the need of strategies to enhance the recognition of the target population of the program activity.

KEYWORDS Health evaluation; Health services; Primary Health Care; Diabetes mellitus; Hypertension.

1 Universidade Estadual de Londrina (UEL) - Londrina (PR), Brasil.

barbara_2887@hotmail.com

2 Universidade Estadual de Londrina (UEL) - Londrina (PR), Brasil.

reginatanno@hotmail.com

3 Universidade Estadual de Londrina (UEL) - Londrina (PR), Brasil.

cordoni@sercomtel.com.br 


\section{Introdução}

As Doenças Crônicas Não Transmissíveis (DCNT), como a Hipertensão Arterial (HA) e Diabetes Mellitus (DM), apresentam número crescente de casos na população, constituem importantes causas de hospitalizações no sistema público de saúde e resultam em sérios problemas de saúde pública. As políticas de enfrentamento desses agravos têm priorizado a organização de serviços pautados na longitudinalidade do cuidado com ênfase na Estratégia Saúde da Família (ESF) (MENDES, 2012).

O controle da hipertensão e do diabetes, no âmbito da Atenção Básica, pode evitar o agravamento e o surgimento de complicações cardiovasculares, com redução do número de internações hospitalares e mortes por essas doenças (SCHMIDT ET AL., 2011). Entretanto, apesar de instituído o padrão de cuidado aos indivíduos com esses agravos, estudos indicam que há um baixo percentual de controle da pressão arterial e glicemia (SOCIEDADE BRASILEIRA DE CARDIOLOGIA; SOCIEDADE BRASILEIRA DE HIPERTENSÃO; SOCIEDADE BRASILEIRA DE NEFROLOGIA, 2010; ROSÁRIO ET AL., 2009).

A detecção precoce dos indivíduos com hipertensão e/ou diabetes pelos serviços da área adscrita é um dos elementos imprescindíveis para o sucesso do controle, pois a partir do reconhecimento a Equipe de Saúde da Família (EqSF) pode realizar o acompanhamento periódico com a finalidade de atender de forma integral às necessidades dos grupos sociais de responsabilidade do território (CAMPOS; MISHIMA, 2005), conforme as diretrizes gerais determinadas pela Política Nacional de Atenção Básica (PNAB) (BRASIL, 2012). Todavia, vários estudos mostram que os serviços de saúde encontram dificuldades na captação da população-alvo que necessita de acompanhamento periódico. Assim sendo, a avaliação constante do desempenho do serviço é fundamental para identificar problemas na atenção e nortear os direcionamentos e aprimorar a qualidade da atenção à saúde (SALA; NEMES; COHEN, 1998).
Frente a tais aspectos, o Ministério da Saúde, em parceria com os estados e municípios, propõe várias iniciativas centradas na qualificação da Atenção Básica, com destaque para o Programa Nacional de Melhoria do Acesso e da Qualidade da Atenção Básica (PMAQ-AB), visando à institucionalização da cultura avaliativa na Atenção Básica no Sistema Único de Saúde (SUS) e a gestão com base no acompanhamento de processos e resultados (BRASIL, 2012).

Assim, considerando-se a importância da avaliação na qualificação da assistência, o objetivo central do estudo é avaliar a cobertura de detecção das pessoas com hipertensão e/ ou diabetes pelas Unidades Básicas de Saúde (UBS) de um município de médio porte.

\section{Material e método}

A pesquisa está inserida no contexto do estudo de base populacional de indivíduos residentes na área urbana 'Doenças Cardiovasculares no Estado do Paraná: mortalidade, perfil de risco, terapia medicamentosa e complicações - VigiCardio', que buscou verificar o perfil de risco cardiovascular de adultos com 40 anos ou mais (SOUZA ET AL., 2013). O VigiCardio foi realizado em Cambé, município da região norte do Paraná com aproximadamente $100 \mathrm{mil}$ habitantes, onde a ESF está implantada em todas as unidades com $87,4 \%$ de cobertura populacional.

$\mathrm{O}$ presente artigo consiste em uma pesquisa avaliativa de base normativa cuja população de estudo foi constituída por 687 indivíduos com hipertensão e ou diabetes identificados entre os 1.180 participantes da amostra do VigiCardio (souza et AL., 2013). Após consulta ao banco de dados do VigiCardio, de posse da listagem contendo dados de identificação e o endereço dos indivíduos com hipertensão e ou diabetes, foram identificados os prontuários e a UBS/ ESF de referência no sistema informatizado 
da Secretaria Municipal de Saúde. Foram excluídos aqueles que faleceram, mudaram de endereço entre a coleta de dados do VigiCardio (primeiro semestre de 2011) e a análise documental (último trimestre de 2012) ou não residiam na área de cobertura da ESF. Após identificação dos indivíduos incluídos no estudo, procedeu-se à coleta de dados na unidade da área de abrangência de cada indivíduo.

Para fins de cálculo da cobertura de detecção das pessoas com hipertensão e/ou diabetes pela UBS da área de abrangência, foi realizada análise do prontuário, consulta ao Sistema de Acompanhamento de Hipertensos e Diabéticos (Sishiperdia) e à ficha de cadastro (Ficha A) do Sistema de Informação da Atenção Básica (Siab). O indivíduo foi considerado detectado pela unidade quando a condição de hipertensão e/ou diabetes, verificada no VigiCardio, estava registrada em pelo menos um desses documentos da UBS da área de abrangência. Dados sobre condições socioeconômicas (sexo, idade, situação conjugal, ocupacional, classificação econômica, escolaridade), de saúde (autopercepção de saúde, classificação de risco cardiovascular individual) e de utilização da UBS foram obtidos no banco de dados do VigiCardio (souza et al., 2013).

Os dados foram duplamente digitados no software Epi Info 2010, versão 3.5.2 (Centers for Disease Control and Prevention, Atlanta, Estados Unidos), tendo sido realizada comparação dos dados e corrigidas as inconsistências. A análise estatística foi realizada com o auxílio do software $S P S S^{\circledast}$, versão 19.0, para Windows (SPSS Inc., Chicago, Estado Unidos).

Para o cálculo da taxa de cobertura, foram considerados no numerador os indivíduos com registro da condição nas fontes consultadas e no denominador a totalidade dos indivíduos considerados com hipertensão e ou diabetes no VigiCardio. Para avaliar a detecção, foi construída uma matriz de indicadores cujos resultados foram comparados ao padrão estabelecido pelo Ministério da Saúde (BRASIL, 2006A; 2006B) e julgados de acordo com os percentuais obtidos: 'excelente' (100-90\%), 'satisfatório' (89-70\%), 'regular' (69-50\%) e 'crítico' (<50\%) (quadro 1).

Quadro 1. Matriz de indicadores para avaliação da cobertura de detecção das pessoas com hipertensão e/ou diabetes pelas unidades básicas do município de Cambé, 2012.

\begin{tabular}{|c|c|c|c|c|c|}
\hline Abordagem & Dimensão & Critério & Indicador & Padrão & Julgamento \\
\hline 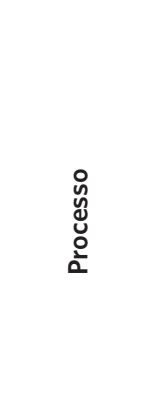 & 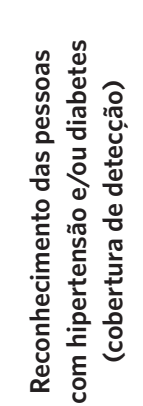 & $\begin{array}{l}\text { 1A. Proporção } \\
\text { de pessoas com } \\
\text { hipertensão } \\
\text { e ou diabetes } \\
\text { detectada }\end{array}$ & 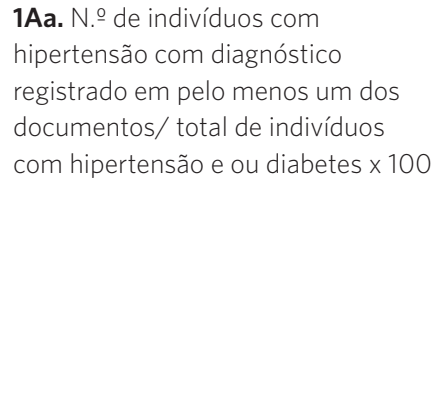 & $\begin{array}{l}\text { 100\% dos } \\
\text { indivíduos } \\
\text { com } \\
\text { hipertensão } \\
\text { e/ou diabetes } \\
\text { reconhecidos } \\
\text { pela UBS } \\
\text { da área de } \\
\text { abrangência }\end{array}$ & $\begin{array}{l}\text { Excelente: } \\
90 \text { a 100\% } \\
\text { Satisfatório: } \\
70 \text { a 89\% } \\
\text { Regular: } \\
50 \text { a 69\% } \\
\text { Crítico: } \\
<50 \%\end{array}$ \\
\hline
\end{tabular}

Fonte: Elaboração própria

Após, procedeu-se a análise dos fatores associados à detecção (variável dependente do estudo). As variáveis independentes foram: sexo, idade, situação conjugal, ocupacional, escolaridade, classificação econômica, autopercepção de saúde, risco cardiovascular individual e utilização da UBS. A variável idade foi agrupada em faixas etárias (40-59 anos e 60 ou 
mais) e a escolaridade em anos completos de estudo (0-7 anos e 8 anos ou mais). A situação conjugal foi categorizada em 'com companheiro' e 'sem companheiro' e a ocupacional em 'trabalha' e 'não trabalha'. A classe econômica dos indivíduos foi analisada utilizando-se os critérios da Associação Brasileira de Empresas e Pesquisas (Abep) (ABEP, 2012), sendo as categorias agrupadas em $\mathrm{A}+\mathrm{B}$ e $\mathrm{C}+\mathrm{D}+\mathrm{E}$. Para verificar a autopercepção de saúde, os indivíduos responderam à seguinte pergunta: 'Como você classifica seu estado de saúde?'. Essa variável foi categorizada em 'Muito bom/ bom' e 'Regular/ ruim/muito ruim'. Para determinar o risco cardiovascular global individual, foi utilizado o escore de Framingham (FRAMINGHAM HEART STUDY, 1998). Após o cálculo, os indivíduos foram considerados com alto, moderado ou baixo risco cardiovascular. A variável utilização do serviço foi categorizada em 'nenhum', 'somente plano' - para os que utilizam somente plano de saúde - e ‘UBS' (com ou sem plano de saúde).

$\mathrm{Na}$ análise bruta, calculou-se a razão entre as taxas de cobertura, valor de p e IC 95\% por meio de regressão de Poisson para verificar a associação entre as variáveis independentes e o desfecho. Para o modelo ajustado, por meio da regressão múltipla de Poisson, foram inseridas as variáveis que apresentaram valor de $\mathrm{p}<0,20$ na análise bruta. Foram consideradas estatisticamente significativas as associações que apresentaram valor de $\mathrm{p}<0,05$.

A presente pesquisa foi aprovada pelo Comitê de Ética em Pesquisa Envolvendo Seres Humanos (CAEE $\mathrm{n}^{\circ}$ : 07831212.9.0000.5231), sendo respeitadas as normas das resoluções 196/96 e 466/12 do Conselho Nacional de Saúde do Ministério da Saúde, em todas as etapas.

\section{Resultados}

Dos 1.180 indivíduos entrevistados no VigiCardio, 687 foram considerados com hipertensão e/ou diabetes pelos critérios estabelecidos. Desses, excluíram-se 55 (8\%) que mudaram de endereço, 7 (1\%) que faleceram e
19 (2,8\%) que não residiam na área de cobertura do PSF. A amostra final foi composta por 606 indivíduos, sendo 78,7\% com hipertensão, 3,5\% com diabetes e 17,8\% com ambas as condições. A maioria era do sexo feminino (54,1\%), tinha de 40-59 anos (59,1\%), possuía de 0 a 7 anos de estudo (69,4\%), vivia com companheiro (73,1\%), pertencia à classe $\mathrm{C}(53,8 \%)$ e exercia atividade remunerada $(53,1 \%)$. Além disso, 52,4\% consideravam sua saúde regular, ruim ou muito ruim; $54,1 \%$ utilizavam somente UBS; 3,0\% não utilizavam qualquer serviço e $41,6 \%$ apresentavam baixo risco cardiovascular individual.

Do total de participantes, verificou-se que em 386 (63,7\%) havia registro da condição em pelo menos um dos documentos analisados. Desses, 305 (79,0\%) tinham o diagnóstico de hipertensão registrado, 17 (4,4\%) o diagnóstico de diabetes e 64 (16,6\%) ambos os diagnósticos. O cálculo dos indicadores dos critérios 1A e 1B (quadro 1), referentes à proporção de pessoas com hipertensão e diabetes, resultou em $63,1 \%$ e $62,8 \%$, respectivamente. Essas proporções indicam que a detecção tanto das pessoas com hipertensão quanto com diabetes pelas UBS foi 'regular'.

Em relação aos fatores associados à detecção, a análise bruta mostrou que a frequência de reconhecimento foi significativamente mais elevada entre as mulheres, indivíduos com 60 anos ou mais, com baixa escolaridade (0-7 anos), que consideraram seu estado de saúde regular, ruim ou muito ruim, que não possuem atividade remunerada e que pertenciam a menor estrato econômico (tabela 1). Quanto à utilização de serviços de saúde, a frequência de reconhecimento foi maior entre os que utilizam a UBS, quando comparada aos que utilizam somente plano de saúde.

Após análise ajustada, permaneceram associadas à detecção pela UBS as variáveis referentes à classe econômica, situação ocupacional e utilização da UBS. Assim, observou-se que a UBS detecta mais aqueles que pertencem à menor classe econômica $(\mathrm{C}, \mathrm{D}$ ou $\mathrm{E}$ ), não exerce atividade remunerada e utilizam a UBS (tabela 1). 
Tabela 1. Características da população estudada, cobertura de detecção (\%), análise bruta e ajustada dos fatores associados à detecção pela Atenção Básica de pessoas com hipertensão e/ou diabetes $(n=606)$. Cambé (PR), 2012

\begin{tabular}{|c|c|c|c|c|c|c|c|c|}
\hline \multirow{3}{*}{ Características } & \multirow{2}{*}{\multicolumn{2}{|c|}{ Total }} & \multirow{2}{*}{\multicolumn{2}{|c|}{$\begin{array}{c}\text { Cobertura } \\
\text { de Detecção }\end{array}$}} & \multicolumn{2}{|c|}{ Análise bruta } & \multicolumn{2}{|c|}{ Análise Ajustada } \\
\hline & & & & & \multirow{2}{*}{$\begin{array}{l}\text { Razão } \\
\text { (IC 95\%) }\end{array}$} & \multirow{2}{*}{$\begin{array}{l}\text { Valor } \\
\text { de } p\end{array}$} & \multirow{2}{*}{$\begin{array}{c}\text { Razão } \\
(\text { IC 95\%) }\end{array}$} & \multirow{2}{*}{$\begin{array}{l}\text { Valor } \\
\text { de } p\end{array}$} \\
\hline & $\mathrm{n}$ & $\%$ & $\mathrm{n}$ & $\%$ & & & & \\
\hline \multicolumn{9}{|l|}{ Sexo } \\
\hline Masculino & 278 & 45,9 & 161 & 57,9 & 1 & \multirow{2}{*}{0,00} & 1 & \multirow{2}{*}{0,57} \\
\hline Feminino & 328 & 54,1 & 225 & 68,6 & $1,18(1,05-1,34)$ & & $1,03(0,91-1,17)$ & \\
\hline \multicolumn{9}{|l|}{ Faixa etária } \\
\hline $40-59$ anos & 358 & 59,1 & 210 & 58,6 & 1 & \multirow{2}{*}{0,00} & 1 & \multirow{2}{*}{0,58} \\
\hline 60 anos ou mais & 248 & 40,9 & 176 & 71,0 & $1,21(1,08-1,36)$ & & $1,03(0,91-1,17)$ & \\
\hline \multicolumn{9}{|l|}{ Escolaridade $^{\star}$} \\
\hline 8 anos ou mais & 185 & 30,6 & 84 & 45,4 & 1 & \multirow{2}{*}{0,00} & 1 & \multirow{2}{*}{0,07} \\
\hline 0 a 7 anos & 420 & 69,4 & 301 & 71,7 & $1,58(1,33-1,87)$ & & $1,16(0,98-1,37)$ & \\
\hline \multicolumn{9}{|l|}{ Classe econômicał } \\
\hline$A+B$ & 204 & 33,8 & 92 & 45,1 & 1 & \multirow{2}{*}{0,00} & 1 & \multirow{2}{*}{0,04} \\
\hline$C+D+E$ & 400 & 66,2 & 292 & 73,0 & $1,62(1,38-1,90)$ & & $1,18(1,00-1,39)$ & \\
\hline \multicolumn{9}{|l|}{ Situação Conjugal } \\
\hline Com companheiro & 443 & 73,1 & 272 & 61,4 & 1 & \multirow{2}{*}{0,06} & 1 & \multirow{2}{*}{0,52} \\
\hline Sem companheiro & 163 & 26,9 & 114 & 69,9 & $1,14(1,01-1,29)$ & & $1,03(0,92-1,16)$ & \\
\hline \multicolumn{9}{|l|}{ Exerce algum trabalho } \\
\hline Sim & 322 & 53,1 & 171 & 53,1 & 1 & \multirow{2}{*}{0,00} & 1 & \multirow{2}{*}{0,00} \\
\hline Não & 284 & 46,9 & 215 & 75,7 & $1,43(1,26-1,61)$ & & $1,26(1,1-1,46)$ & \\
\hline \multicolumn{9}{|l|}{ Autopercepção de Saúde } \\
\hline Muito boa/ boa & 288 & 47,6 & 162 & 56,2 & 1 & \multirow{2}{*}{0,00} & 1 & 015 \\
\hline Regular/ ruim/ muito ruim & 317 & 52,4 & 224 & 70,7 & $1,26(1,11-1,42)$ & & $1,08(0,96-1,22)$ & \\
\hline $\begin{array}{l}\text { Utilização dos serviços de } \\
\text { saúdeł }\end{array}$ & & & & & & & & \\
\hline Somente plano & 80 & 13,3 & 18 & 22,5 & 1 & & 1 & \\
\hline UBS & 505 & 83,7 & 360 & 71,3 & $3,17(2,10-4,78)$ & 0,00 & $2,68(1,75-4,09)$ & 0,00 \\
\hline Nenhum & 18 & 3,0 & 5 & 27.7 & $1,23(0,53-2,88)$ & 0,86 & $1,19(0,52-2,69)$ & 0,67 \\
\hline Risco cardiovascular§ & & & & & & & & \\
\hline Baixo risco & 199 & 41,6 & 127 & 63,8 & 1 & & - & - \\
\hline Risco moderado & 195 & 40,8 & 125 & 64,1 & $1,00(0,87-1,16)$ & 0,96 & - & - \\
\hline Alto risco & 84 & 17,6 & 59 & 70,2 & $1,10(0,92-1,31)$ & 0,36 & - & - \\
\hline
\end{tabular}

Fonte: Elaboração própria

*Excluído 1 caso; †Excluídos 2 casos; + Excluídos 3 casos; §Excluídos 128 casos

\section{Discussão}

Os resultados obtidos por meio dos cálculos dos indicadores dos critérios 1A- 'Proporção de indivíduos com hipertensão detectados' e 1B- 'Proporção de indivíduos com diabetes detectados' mostraram que as UBS detectaram $63,1 \%$ das pessoas com hipertensão e $62,8 \%$ das pessoas com diabetes.

Apesar de a meta pactuada ser $100 \%$, estudos apontam dificuldades na detecção desses indivíduos. A detecção 'regular' dos 
indivíduos com hipertensão e/ou diabetes pelas unidades de saúde pode estar relacionada à dificuldade de operacionalização das ações de detecção, já aventada por Sala, Nemes e Cohen (1998) no estudo realizado em uma unidade de saúde do município de São Paulo, e mais recentemente por Louvison (2011), em uma pesquisa longitudinal, na qual foram reentrevistados idosos da comunidade, em 2006, no município de São Paulo. Entre as dificuldades na detecção pelos serviços de saúde, o autor destaca a identificação precoce, cuja fragilidade advém não só porque os idosos não procuraram o serviço, mas também porque o serviço não estava atento ao momento oportuno do diagnóstico (LOUVISON, 2011).

Avaliação normativa realizada com uma amostra representativa de 73 EqSF no município de Recife, que analisou o grau de implantação das ações de atenção ao diabetes mellitus, também mostrou que um dos principais problemas foi a fragilidade das ações de diagnóstico precoce. Entre os indicadores que não foram implantados, conforme Pereira (2007), encontrava-se o rastreamento do diabetes.

No presente trabalho, as coberturas de detecção chamam a atenção pelo fato de a ESF se constituir no eixo estruturante no município e encontrar-se implantada em todas as UBS com cobertura populacional de $87,4 \%$. O principal foco das EqSF é a prestação de assistência integral, contínua, com resolutividade e qualidade às necessidades da população adstrita (MENDES, 2012). Além disso, esse modelo de atenção pauta-se na ação programática, que é uma lógica compreendida como de importância primordial na continuidade da atenção integral (ROSA FILHO, 2006).

Todavia, não foi encontrado nenhum artigo no qual estudo de base populacional tenha constituído a linha de base na identificação de pessoas da comunidade com hipertensão e ou diabetes para, posteriormente, verificar em que medida os serviços estruturados na lógica programática têm captado a população-alvo. No presente estudo, apesar da cobertura obtida apresentar-se aquém da meta pactuada, a maior frequência de detecção das pessoas economicamente desfavorecidas sugere avanços importantes no município estudado, pois indica maior alcance do SUS como política pública de inclusão de pessoas com menor acesso às ações e serviços de saúde. Desse modo, é possível supor que, de certo modo, a ESF tem contribuído para a redução das iniquidades na atenção.

A maior cobertura, já esperada, dos indivíduos que utilizam a UBS reforça que a identificação ainda ocorre principalmente no serviço entre aqueles que constituem demanda espontânea do sistema. Por esse lado, pode-se considerar ponto positivo o fato de estarem sendo detectados os indivíduos que utilizam a UBS. Por outro, indica que há necessidade de aprimorar as ações para que os serviços sejam capazes de captar também as pessoas que não utilizam a UBS, especialmente as que não dispõem de nenhum outro serviço alternativo ao SUS. Vale destacar que a taxa de detecção foi de apenas $27,7 \%$ entre as pessoas que referiram utilizar nenhum serviço de saúde. Embora a diferença não tenha sido estatisticamente significativa, provavelmente devido à casuística reduzida, esses resultados sugerem existência de problemas tradicionais relacionados às barreiras organizacionais e de funcionamento. Segundo Ribeiro et al. (2006), para boa parte da população usuária SUS, os hospitais e prontos-socorros ainda são importantes portas de entrada para assistência à saúde.

Reforça tal suposição a taxa de detecção mais elevada entre aqueles sem atividade ocupacional verificada na presente investigação, pois em pesquisa realizada por Pedraza e Costa (2014) constatou-se que fatores organizacionais, como o tempo de espera para marcar uma consulta e para o atendimento em geral, constituem importantes barreiras de acesso e utilização do serviço, que se estendem a 
outros aspectos assistenciais apontados por diferentes estudos, como o sistema de marcação de consultas (CUNHA; VIEIRA-DA-SILVA, 2010), a obtenção de medicamentos, a realização de exames e o acesso à assistência especializada (MENDES ET AL., 2012).

Ressalta-se que, embora o PSF tenha avançado de programa para estratégia com vistas a superar a insuficiência dos arranjos organizacionais, falência das práticas profissionais e representar uma proposta de reorganização da Atenção Básica como eixo de reorientação do modelo assistencial -, a universalização da atenção ainda não está satisfatória (MENDES, 2012). Apesar de estudos apontarem que os desempenhos das unidades com PSF implantado são melhores quando comparados às UBS com modelo tradicional (FACCHINI ET AL., 2006), os resultados do presente estudo permitem aventar que a proposta do modelo centrado na saúde da família ainda não foi incorporada pelos profissionais, uma vez que se supõe que os indivíduos detectados são aqueles que utilizam a UBS.

Ainda que um dos objetivos dos protocolos de saúde do adulto com ênfase na hipertensão e diabetes seja a busca, a detecção e inserção no programa de atendimento de todos os portadores da doença (BRASIL, 2006A; 2006B), os resultados mostram que há necessidade de avançar no processo de institucionalização da ESF nos diferentes espaços e na organização do serviço.

Avanços nos fatores organizacionais do serviço, como a acessibilidade organizacional, poderiam melhorar a utilização e, por consequência, a cobertura de detecção. Nesse sentido, a abertura da UBS em horários alternativos facilitaria a utilização por parte dos trabalhadores que não procuram serviços de saúde da rede básica devido à incompatibilidade temporal (CUNHA; VIEIRADA-SILVA, 2010). Além disso, a capacitação da equipe para implementar ações educativas junto à população no sentido de difundir informações sobre os principais agravos à saúde, com destaque nas estratégias de promoção da saúde e controle dos fatores de risco das DCNT, poderia ampliar as possibilidades de autocuidado e deliberação dos indivíduos sobre os diversos determinantes de sua saúde.

Há necessidade, também, de maiores investimentos no processo de captação, seja mediante busca ativa nos domicílios pelas EqSF, aconselhamento dos que desconhecem a sua condição para procurarem serviços de saúde ou, ainda, no rastreamento dos casos pelos profissionais de saúde em todas as oportunidades de atenção.

Além dos aspectos mencionados, frente aos resultados obtidos, há que se considerar como limitação do presente trabalho o fato de não ter sido possível a discriminação dos casos que podem corresponder a indivíduos sem diagnóstico de hipertensão e/ou diabetes, pois no estudo de base populacional foram adotados critérios de rastreamento, com alta sensibilidade. Dessa forma, é possível que a prevalência de hipertensão e diabetes esteja, em certa medida, superestimada, com consequente subestimativa da taxa de cobertura de detecção.

\section{Conclusões}

Os resultados sobre a detecção regular das pessoas com hipertensão e/ou diabetes pela Atenção Básica e menor cobertura dos indivíduos que não utilizam a UBS ou com atividade ocupacional reiteram a importância da pesquisa avaliativa na reflexão sobre o alcance da população-alvo dos serviços e sobre as principais estratégias vigentes de detecção e de atenção aos grupos programáticos. Considerando-se que os programas de controle da hipertensão são os mais estruturados na atenção primária à saúde, com propostas iniciais de implantação há quatro décadas, é possível inferir que a situação observada seja traçadora da qualidade da atenção prestada pelas UBS 
no enfrentamento de outras DCNT e a realidade do município estudado, com ampla cobertura do PSF, também se aproxime à de outras localidades do País. Reforçam, desse modo, a necessidade de investimentos que aprimorem os mecanismos de captação que ultrapassem os limites

\section{Referências}

ABEP, Associação Brasileira de Empresas de Pesquisa. CCEB: Critério Brasil. 2012. Disponível em: <http:// www.abep.org/novo/Content.aspx?SectionID=84>. Acesso em: 20 jul. 2012.

BRASIL. Ministério da Saúde. Secretaria de Atenção à Saúde. Departamento de Atenção Básica. Hipertensão arterial sistêmica para o sistema único de saúde. Brasília, DF: Ministério da saúde, 2006a. 53 p. (Cadernos de Atenção Básica n. 15).

Ministério da Saúde. Secretaria de Atenção à Saúde. Departamento de Atenção Básica. Diabetes Mellitus. Brasília, DF: Ministério da Saúde, 2006b. 56 p. (Cadernos de Atenção Básica n. 16).

Ministério da Saúde. Secretaria de Atenção à Saúde. Departamento de Atenção Básica. Política Nacional de Atenção Básica. 4. ed. Brasília, DF: Ministério da Saúde, 2012. 110 p.

CAMPOS, C. M. S.; MISHIMA, S. M. Necessidades de saúde pela voz da sociedade civil e do Estado. Cadernos de Saúde Pública; Rio de Janeiro, v. 21, n. 4, p. 12601268, jul./ago. 2005.

CUNHA, A. B. O.; VIEIRA-DA-SILVA, L. M. Acessibilidade aos serviços de saúde em um município do Estado da Bahia, Brasil, em gestão plena do sistema. Cadernos de Saúde Pública; Rio de Janeiro, v. 26, n. 4, p. 725-737, abr. 2010.

FACCHINI, L. A. et al. Desempenho do PSF no Sul e no Nordeste do Brasil: avaliação institucional e epidemiológica da Atenção Básica à Saúde. Ciência e Saúde Coletiva; Rio de Janeiro, v. 1l, n. 3, p. 669-681, jul./set. 2006. tradicionalmente instituídos de atuação do serviço de saúde, o que envolve mudança cultural na concepção do sistema de saúde brasileiro em busca da concretização dos princípios do SUS, com ampla aceitação e incorporação pelo sistema, profissionais e população.
FRAMINGHAM HEART STUDY. A Project of the National Heart, Lung and Blood Institute and Boston University. Coronary Heart Disease (10-years risk). 1998. Disponível em: <http://www.framinghamheartstudy.org/risk/coronary.html\#tab3 >. Acesso em: 26 out. 2012.

LOUVISON, M. C. P. Avaliação da atenção às condições crônicas em idosos: hipertensão arterial sistêmica e diabetes mellitus como condições traçadoras. 2011. Tese (Doutorado em Saúde Pública) - Faculdade de Saúde Pública da Universidade de São Paulo, São Paulo, 2011.

MENDES, E. V. O cuidado das condições crônicas na atenção primária à saúde: o imperativo da consolidação da estratégia da saúde da família. Brasília, DF: Organização Pan Americana da Saúde, 2012, 512 p.

MENDES, A. C. G. et al. Acessibilidade aos serviços básicos de saúde: um caminho ainda a percorrer. Ciência e saúde coletiva, Rio de Janeiro, v. 17, n. 11, p. 2903-2912, 2012.

PEDRAZA, D. F.; COSTA, G. M. C. Acessibilidade aos serviços públicos de saúde: uma visão dos usuários da Estratégia Saúde da Família. Revista electrônica trimestral de Enfermaria; n. 33, p. 279-91, 2014.

PEREIRA, P. M. H. Avaliação da atenção básica para o diabetes mellitus na Estratégia de Saúde da Família. 2007. Dissertação (Mestrado em Saúde Pública) Fundação Oswaldo Cruz, Centro de Pesquisa Aggeu Magalhães, Recife, 2007.

RIBEIRO, M. C. S. A. et al. Perfil sociodemográfico e padrão de utilização de serviços de saúde para usuários 
e não usuários do SUS - PNAD 2003. Ciência e saúde coletiva, Rio de Janeiro, v. 11, n. 4, p. 1011-22, 2006.

ROSA FILHO, L. A. Determinantes e efeitos da continuidade na atenção à saúde: estudo de base populacional em Pelotas, RS. 2006. Dissertação (Mestrado em Epidemiologia) - Universidade Estadual de Pelotas, Pelotas, 2006.

ROSÁRIO, E. M. et al. Prevalência, controle e tratamento da hipertensão arterial sistêmica em Nobres MT. Arquivos Brasileiro de Cardiologia, Rio de Janeiro, v. 93, n. 6, p. 671-678, 2009.

SALA, A.; NEMES, M. I. B.; COHEN, D. D. Metodologia de avaliação do trabalho na atenção primária à saúde. Cadernos de Saúde Pública; Rio de Janeiro, v. 14, n. 4, p. 741-751, out./dez. 1998.

SOCIEDADE BRASILEIRA DE CARDIOLOGIA; SOCIEDADE BRASILEIRA DE HIPERTENSÃO;
SOCIEDADE BRASILEIRA DE NEFROLOGIA.

VI Diretrizes Brasileiras de Hipertensão. Arquivos

Brasileiro de Cardiologia, Rio de Janeiro, v. 95. supl. 1, p.

$1-51,2010$.

SCHMIDT, M. I. et al. Saúde no Brasil 4: Doenças crônicas não trasnmissíveis no Brasil: carga e desafios atuais. Lancet, p. 61-74, maio, 2011. Disponível em: <http:// www.abc.org.br/IMG/pdf/doc-574.pdf>. Acesso em: 30 mar. 2014

SOUZA, R. K. T. et al. Prevalência de fatores de risco cardiovascular em pessoas com 40 anos ou mais de idade, em Cambé, Paraná (2011): estudo de base populacional. Epidemiologia Serviços e Saúde; Brasília, DF, v. 22, n. 3, jul./set. 2013.

Recebido para publicação em março de 2014

Versão final em agosto de 2014

Conflito de interesse: inexistente

Suporte financeiro: não houve 